\title{
HANDLING OF PREPRINTS
}

\author{
M. E. Gómez \\ European Southern Observatory \\ Library La Silla \\ Casilla 19001 - Santiago 19 \\ Chile
}

\begin{abstract}
At La Silla, we receive on average between 90 and 130 preprints a month from institutions all over the world. The preprints are extensively used by our staff as well as visiting astronomers who come to perform observations at La Silla. Most of the preprints are received as donations, others are requested directly from the publishing institute.
\end{abstract}

The European Southern Observatory has among other publications, its ESO Scientific Preprints. At La Silla, the library does not deal with the distribution of these preprints, since this is made directly from ESO in Garching, FRG.

As soon as the preprints arrive to the library, they are stamped, included in a list to be distributed at the end of the month, and displayed on the shelf at the Astronomy Lounge. The Astronomy Lounge is a special room dedicated to informal astronomical discussions. It contains shelves where preprints, and the latest main astronomical journals are displayed. In this inspiring atmosphere, astronomers may meet to discuss about their last research and get ideas for new projects.

The importance of the preprints was amply proved after the SN1987a was discovered. For example, the results from the research made at ESO was made available to a larger public in the form of an ESO Scientific Preprint containing several papers. Since preprints normally appear several months earlier than the printed version of the papers, many astronomers receive first news about advances in their field from such preprints.

The shelf for the preprints has 96 slanted compartments, one for every preprint, which are placed vertically from top to down. If the space is not sufficient, the preprints published by the same institute are assigned to the same compartment, leaving the last one on top.

The preprints are on display for one month. At the end of the month, they are moved to a plastic cabinet with drawers which contain the old preprints for one year separated by months. The old preprints from the previous year for the same month 
are removed and distributed to anyone interested.

Before this, it is checked if they have been published in a journal or book not available at the library. If this is the case, they are kept under the publishing institution.

The ESO Scientific Preprint series published by ESO is bound and kept as observatory publication.

As a way to inform interested users about the preprints that have arrived, a monthly list ordered by author is also distributed as well as displayed in the library. This list also includes the observatory publications and books received during the last month.

The information in this list includes:

- author

- home institution of the first author

- title

- name of the publishing institution, if the home institute of the first author is different

- name of the series, as well as number, month and year of the publication

While the preprints continue to be an important source for the latest astronomical news, the importance of the reprints has diminished. Most of the reprints we receive are contributions from astronomical observatories, that we keep only if we do not have the original publication. Others are directly requested from the authors.

However, with the existence of modern photocopying machines, reprints are not used anymore as a way of getting a personal copy of the publication you are interested in. Maybe in the near future the importance of also the preprints will diminish due to modern electronic distribution systems. Already now, some astronomers are sending their latest papers to interested persons, via electronic mail. 